\title{
Thesenförmige Zusammenfassung
}

1. Das IASB strebt mithilfe des Rahmenkonzepts eine deduktive Ermittlung von Rechnungslegungsvorschriften an. Die im Rahmenkonzept festgelegte Zwecksetzung sowie die qualitativen Anforderungen dienen dabei als Deduktionsgrundlage zur Ableitung konsistenter Regelungen auf Einzelstandardebene. Der Zweck der Entscheidungsnützlichkeit und die diesem untergeordnete Rechenschaftsfunktion sowie die Anforderung der Relevanz und der glaubwürdigen Darstellung erlauben jedoch unterschiedliche Konkretisierungsmöglichkeiten. Auch die in den vergangenen Jahren erfolgte Rahmenkonzeptüberarbeitung schränkt aufgrund der zeitgleichen Entwicklung neuer Standards und der fehlenden Anpassung alter Standards die Funktion des Rahmenkonzepts als normative Grundlage der Standardsetzung ein.

2. Die Standardsetzung des IASB weist zudem Anknüpfungspunkte einer induktiven Ermittlung von Rechnungslegungsvorschriften auf, die sich durch eine Orientierung an der Rechnungslegungspraxis und einzelfallspezifische Regelungen auszeichnet. Das Rahmenkonzept ermöglicht durch die faktische Berücksichtigung von Unternehmensaktivitäten sowie insbesondere aufgrund der in jedem Standardsetzungsprozess verpflichtenden KostenNutzen-Abwägung induktiv geprägte Regelungsentscheidungen. Auch die Aufnahme von Vereinfachungsreglungen auf Einzelstandardebene sowie die Entwicklung branchenindividueller Standards entspricht einer praxisorientierten Regelungsentwicklung.

3. Anstelle der Einordnung der IFRS-Standardsetzung als deduktiv oder induktiv ist die Ermittlung von Rechnungslegungsvorschriften als rekursiver Normermittlungsprozess zu charakterisieren. Im Sinne des hermeneutischen 
Ansatzes kann die Regelungsentwicklung vor dem Hintergrund einer deduktiven Ableitung aus dem Rahmenkonzept sowie einer gleichzeitig induktiven Erschließung der Bilanzierungspraxis erklärt werden. Dieser rekursive Verstehensprozess spiegelt sich im Due Process durch den kontinuierlichen Austausch zwischen dem IASB und den am Standardsetzungsprozess Beteiligten wider. Der wechselseitige Einfluss von Standardsetzung und Standardanwendung stellt demnach eine wesentliche Determinante der Normermittlung dar.

4. Die IFRS führen als kodifizierte Rechnungslegungsstandards zur Regulierung der Rechnungslegung, weshalb die Standardsetzung nicht nur eine technische Aufgabe, sondern auch einen politischen Prozess darstellt. Die vom IASB entwickelten und noch nicht in EU-Recht übernommenen Rechnungslegungsstandards erfordern aufgrund der privatrechtlichen Organisation der IFRS Foundation respektive dem IASB die Akzeptanz der Betroffenen. Die Legitimation wird sowohl input-orientiert durch die Partizipationsmöglichkeiten im Rahmen des öffentlichen Standardsetzungsprozesses als auch output-orientiert durch die markteffiziente Ausrichtung der durch Experten erstellten Standards angestrebt. Der Due Process ermöglicht Interessengruppen eine Einflussnahme, wobei besonders bei Unternehmen als Anwender der Regelungen ein hoher Anreiz zur Teilnahme besteht. Um einer einseitigen Interessenberücksichtigung entgegenzuwirken, bedarf es im Rahmen der Regelungsentwicklung daher einer Interessenabwägung in Form einer Kompromissfindung.

5. Die Legitimationsfunktion des Due Process liegt in der Sicherstellung eines diskursiven Standardsetzungsprozesses. Neben den Partizipationsmöglichkeiten im Rahmen der Teilnahme in Beratungsgremien und Feldstudien sind als verpflichtender Verfahrensbestandteil sowohl bei der Agendasetzung als auch in den Projektphasen der Forschung, Standardsetzung sowie Implementierung und Instandhaltung öffentliche Konsultationen vorgesehen. Die Interessengruppen kommentieren die Regelungsvorschläge durch Stellungnahmen und können auf diese Weise Einfluss auf die Regelungsentwicklung nehmen. Das IASB erlangt durch die Stellungnahmen standardsetzungsrelevante Informationen und liefert in den Basis for Conclusions eine Regelungsbegründung. Die Entwicklung von Rechnungslegungsvorschriften und die Effizienz sowie die Dauer eines Standardsetzungsprozesses wird somit nicht nur durch die Kompromiss-, sondern auch durch die Kommunikationsfähigkeit des IASB und der beteiligten Akteure bedingt.

6. Der Kompromisscharakter der Regelungsvorschriften fördert Ermessensspielräume, die im Rahmen der Standardanwendung von Unternehmen zu 
konkretisieren sind. Auch bei der Schließung von Regelungslücken agieren Unternehmen bei der Entwicklung sachgerechter Bilanzierungslösungen unter Berücksichtigung ähnlicher Standards, des Rahmenkonzepts sowie von Rechnungslegungsstandards anderer Standardsetzer und anerkannter Branchenpraktiken - als Normentwickler. Die Standardanwendung beinhaltet insofern eine rechtsfortbildende Komponente. Sie kann einerseits Anstoß zu neuen Standardsetzungsverfahren geben, andererseits jedoch auch einen Wandel in der Standardsetzung beschränken, sofern eine im Vergleich zur etablierten Bilanzierungspraxis konzeptionelle Neuausrichtung mit einer im Sinne der Regelanwendbarkeit an der Bilanzierungspraxis orientierten Ausgestaltung der Rechnungslegungsvorschriften konfligiert.

7. Im Sinne des konstruktivistischen Forschungsansatzes kann der Einfluss von Unternehmen auf die Regelungsentwicklung durch einen konstitutiven Erklärungsansatz unter Berücksichtigung diskurstheoretischer Annahmen analysiert werden. Nicht nur die im Standardsetzungsprozess diskutierten Regelungsvorschläge und Ausgestaltungsmöglichkeiten als inhaltliche Zielrichtung, sondern auch die gewählte Rhetorik wird dabei für die Anschlussfähigkeit und Etablierung einer Regelungsvorschrift als maßgeblich erachtet. Sowohl Stellungnahmen als auch Standardentwürfe sind öffentlich verfügbar und stellen daher eine objektive Datenbasis dar. Während die Standardentwürfe Auskunft über die Ansichten des IASB ermöglichen, zeigen die Unternehmensstellungnahmen die Ansichten der Bilanzierungspraxis auf, wodurch sich dem rekursiven Verhältnis zwischen Standardsetzung und Standardanwendung genähert werden kann.

8. Die Entwicklung von Regelungsvorschriften zur Bilanzierung von Mehrkomponentengeschäften war als eines der Standardsetzungsziele von IFRS 15 zugleich einer der wesentlichen Gründe des IASB für die Überarbeitung der Standards zur Umsatzerfassung. Die für die Abgrenzung eines Mehrkomponentengeschäfts relevanten Regelungen des IFRS 15 zur Vertragszusammenfassung, zur Portfolio-Option, zur Aufteilung von Leistungsverpflichtungen anhand des Kriteriums der Eigenständigkeit sowie zur Aufteilung des Transaktionspreises auf Basis der relativen Einzelveräußerungspreise sind durch umfangreiche Kriterien und Erläuterungen in Form von Indikatoren und Beispielen gekennzeichnet. Trotz dieses Regelungskonglomerats eröffnen sich jedoch Ermessensspielräume, die wiederum eine Konkretisierung durch die Unternehmen im Rahmen der Standardanwendung erfordern.

9. Für die Analyse des Einflusses von Unternehmen auf die Regelungsentwicklung eignen sich inhaltsanalytische Verfahren, die durch eine systematische Kategorisierung von Texten eine von der Forschungsfrage geleitete Erfassung 
sowie im Fall eines qualitativen Vorgehens eine Bedeutungserschließung relevanter Inhalte erlauben. Die für die Untersuchung der Einflussnahme von Interessengruppen im Rahmen von Rechnungslegungsstandardsetzungsprozessen durchgeführten Studien fokussieren sich auf die Beteiligung der Interessengruppen, auf ihre inhaltlichen Positionen sowie auf den Erfolg ihrer Teilnahme. Mithilfe des Ansatzes der argumentativen Diskursanalyse können zudem diskursive Strukturen im Standardsetzungsprozess offengelegt werden, indem die von den beteiligten Akteuren verwendeten charakteristischen story-lines rekonstruiert werden, was Rückschlüsse auf die Etablierung bestimmter Bedeutungsgehalte und die Akzeptanz von Regelungsvorschriften ermöglicht.

10. Die Durchführung der qualitativen Inhaltsanalyse für die Regelungen zur Umsatzerfassung aus Mehrkomponentengeschäften erfolgte durch die Codierung der Unternehmensstellungnahmen und Standardentwürfe zu IFRS 15 anhand theoriebasierter und thematisch am Material gebildeter Kategorien, die die inhaltliche und rhetorische Dimension des Standardsetzungsprozesses umfassen. Unter der Hauptkategorie der inhaltlichen Zielrichtung wurden der Inhalt und die Form der Regelungsvorschriften, mithin sowohl die während des Standardsetzungsprozesses diskutierten Bilanzierungsalternativen als auch deren Umsetzungsmöglichkeiten, erfasst. Die Überzeugungsarten Ethos, Logos und Pathos, die der Erzeugung von Glaubwürdigkeit, Emotionen und Rationalität dienen, sowie die rhetorischen Stilmittel wurden unter der Hauptkategorie der Rhetorik subsumiert. Die inhaltlich strukturierende Codierung bildete die Grundlage für die Identifikation der im Standardsetzungsprozess genutzten story-lines.

11. Die Verteilung der Unternehmensstellungnahmen im Standardsetzungsprozess von IFRS 15 zeigt eine höhere Beteiligung von Unternehmen in späteren Standardentwurfsphasen als im Discussion Paper und war im Exposure Draft am höchsten; die relative Beteiligung von Unternehmen im Vergleich zu anderen Interessengruppen war im Verlauf des Standardsetzungsprozesses mit einem Anteil von etwa $30 \%$ hingegen überwiegend konstant. Die Mehrheit der beteiligten Unternehmen war US-amerikanischer Herkunft, was in Zusammenhang mit der Entwicklung des Standards als Konvergenzprojekt mit dem FASB steht. Die Beteiligung europäischer Unternehmen lag bei $16,99 \%$, wohingegen die Beteiligung von Unternehmen zentral- und südamerikanischer Herkunft mit unter $1 \%$ am geringsten war. Die Branchenverteilung zeigt mit fast $50 \%$ eine Dominanz von Stellungnahmen von Unternehmen aus der Fertigungsindustrie. 
12. Der Einfluss von Unternehmen auf die inhaltliche Zielrichtung wurde anhand der Häufigkeit der kommentierten Bilanzierungsalternativen auf Unternehmensebene und der Kookkurrenz der Umsetzungsmöglichkeiten unter Berücksichtigung der in den Standardentwürfen von den Standardsetzern begründeten Regelungsvorschriften beurteilt. Die Analyse zeigt einerseits, dass Unternehmen bei Änderungen eines Standards, der sich in der Bilanzierungspraxis etabliert hat, die Beibehaltung existierender Regelungsvorschriften forcieren, mithin einer konzeptionellen Neuausrichtung entgegenwirken. Andererseits stellen die im Laufe des Standardsetzungsprozesses von den Standardsetzern - teilweise explizit unter Verweis auf die Unternehmenskommentierungen - vorgenommenen Anpassungen der in Frage stehenden Bilanzierungsalternativen eine Annäherung an die vorherige Bilanzierungspraxis bzw. eine Ausgestaltung zugunsten der Anwendbarkeit einer Rechnungslegungsregelung dar.

13. Die Einflussnahme der Unternehmen resultierte in Kompromisslösungen, wie etwa der Portfolio-Option, dem Indikator der wechselseitigen Beziehung zwischen Gütern und Dienstleistungen zur Aufteilung von Leistungsverpflichtungen oder dem Residualwertansatz als Methode zur Schätzung des Einzelveräußerungspreises, durch die der konzeptionelle Anspruch eines branchenübergreifenden und vertragsbasierten Umsatzerfassungsmodells gewährleistet, gleichzeitig jedoch bilanzierungspraktische Anwendungsmöglichkeiten geschaffen wurden. Die Analyseergebnisse verdeutlichen daher das rekursive Verhältnis zwischen Standardsetzung und Standardanwendung.

14. Durch die Teilnahme am Standardsetzungsprozess können sich Unternehmen mit den geplanten Regelungsvorschriften frühzeitig auseinandersetzen und durch Stellungnahmen zu einer anwendungsorientierten Konkretisierung der Regelungen beitragen. Das IASB als Standardsetzer kann durch die Berücksichtigung der Stellungnahmen von Unternehmen die Anwendbarkeit der Regelungen sicherstellen und durch kompromissfähige Bilanzierungslösungen dem Legitimationserfordernis Rechnung tragen. Der Einfluss von Unternehmen auf den IFRS-Standardsetzungsprozess bedingt jedoch auch den konzeptionellen Wandel von Regelungsvorschriften, der insoweit in den Grenzen der Standardanwendung verläuft.

15. Der Einfluss der rhetorischen Strategie auf die Regelungsentwicklung wurde anhand der Häufigkeit und Kookkurrenz der verwendeten Überzeugungsarten und rhetorischen Stilmittel auf Ebene der Codier-Häufigkeiten beurteilt. Die Analyse legt nahe, dass die Überzeugungskraft von Emotionen von den Unternehmen und den Standardsetzern als gering angenommen wird. 
Sofern Pathos verwendet wurde, erfolgte dies überwiegend in Zusammenhang mit der Anwendbarkeit einer Regelung. Während Unternehmen im Standardsetzungsverfahren in ihren Stellungnahmen tendenziell häufiger die Überzeugungsart Ethos nutzten, tendierten die Standardsetzer zur Überzeugung durch Logos.

16. Im Hinblick auf die Erzeugung von Rationalität konnten für Unternehmen die Ergebnisse anderer Lobbying-Studien bestätigt werden, wonach zwar sowohl konzeptionelle als auch ökonomische Gründe verwendet wurden, jedoch eine leichte Tendenz zur Begründung anhand ökonomischer Auswirkungen besteht. Die Standardsetzer verwendeten in Einklang mit der angestrebten rahmenkonzeptbasierten Standardsetzung überwiegend konzeptionelle Gründe, bezogen sich indes auch auf ökonomische Gründe, was wiederum die Berücksichtigung der Standardanwendbarkeit im Rahmen der Standardsetzung verdeutlicht.

17. Die Erzeugung von Glaubwürdigkeit diente den Unternehmen zur Überzeugung der Standardsetzer von ihrer Standardsetzungskompetenz, indem die eigene Expertise betont sowie Respekt gegenüber den Regelungsvorschlägen der Standardsetzer und ein geteiltes Verständnis der Standardsetzungsziele vermittelt wurden. Die Verwendung von Ethos äußerte sich bei den Standardsetzern hingegen in der Vermittlung von Respekt gegenüber den Stellungnehmern durch den Einbezug der Kommentierungen und der Betonung der eigenen Standardsetzungskompetenz, um die am Standardsetzungsprozess Beteiligten von der Legitimation ihrer Standardsetzungsbefugnis zu überzeugen.

18. Der Einsatz rhetorischer Stilmittel erfolgte bei allen Überzeugungsarten. Durch die Verwendung von Übertreibungen in Zusammenhang mit Pathos wurde die Vermittlung der Unmöglichkeit der Anwendbarkeit einer diskutierten Bilanzierungsalternative bezweckt. Die Unternehmen und die Standardsetzer verwendeten zudem häufig die User-Metonymie und die Antithese. Während der Verweis auf die Nutzer überwiegend in Zusammenhang mit konzeptionellen Gründen erfolgte und folglich die Überzeugung durch Logos stärkte, zielte der Einsatz von Antithesen auf die Vermittlung von Respekt und demzufolge auf die Vermittlung von Ethos. Die Standardsetzer nutzen zudem die Most/Some-Metonymie, um die Berücksichtigung der Stellungnahmen zu betonen, was wiederum der Erzeugung von Glaubwürdigkeit diente.

19. Unter Anwendung der argumentativen Diskursanalyse wurden bei den untersuchten Bilanzierungsalternativen im Standardsetzungsprozess von IFRS 15 zwei zentrale story-lines identifiziert, die als diskursiv etablierte Narrationen 
die Standardsetzung strukturieren. Unter der „user needs“ story-line werden die Bedürfnisse und Interessen der Rechnungslegungsnutzer und unter der „economic reality" story-line wird die Darstellung der ökonomischen Realität einer Transaktion als Rechtfertigung für die Angemessenheit einer Regelung konstituiert. Sowohl die Nutzerbedürfnisse als auch die ökonomische Realität werden von den Unternehmen und den Standardsetzern indes erst im Rahmen der diskursiven Auseinandersetzung konstruiert, gleichzeitig als rhetorische Konstrukte bei der Regelungsentwicklung verwendet. Beide story-lines verdeutlichen, dass sich der Standardsetzungsprozess in den Grenzen diskursiver Strukturen bewegt, die sich das IASB und die Unternehmen als am Standardsetzungsprozess Beteiligte vor dem Hintergrund ihrer für den Wandel von Regelungsvorschriften determinierenden Wirkung vergegenwärtigen sollten.

Open Access Dieses Kapitel wird unter der Creative Commons Namensnennung 4.0 International Lizenz (http://creativecommons.org/licenses/by/4.0/deed.de) veröffentlicht, welche die Nutzung, Vervielfältigung, Bearbeitung, Verbreitung und Wiedergabe in jeglichem Medium und Format erlaubt, sofern Sie den/die ursprünglichen Autor(en) und die Quelle ordnungsgemäß nennen, einen Link zur Creative Commons Lizenz beifügen und angeben, ob Änderungen vorgenommen wurden.

Die in diesem Kapitel enthaltenen Bilder und sonstiges Drittmaterial unterliegen ebenfalls der genannten Creative Commons Lizenz, sofern sich aus der Abbildungslegende nichts anderes ergibt. Sofern das betreffende Material nicht unter der genannten Creative Commons Lizenz steht und die betreffende Handlung nicht nach gesetzlichen Vorschriften erlaubt ist, ist für die oben aufgeführten Weiterverwendungen des Materials die Einwilligung des jeweiligen Rechteinhabers einzuholen.

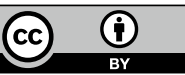

